\title{
Mathematical Teaching and Learning Environment Mediated by ICT
}

\author{
Giampaolo Chiappini, Bettina Pedemonte and Elisabetta Robotti \\ Istituto per le Tecnologie Didattiche - Consiglio Nazionale delle Ricerche, \\ chiappini@ima.ge.cnr.it
}

\begin{abstract}
This paper presents a theoretical model we draw on when designing and developing ICT-based systems for mathematics teaching and learning which falls within the cultural-historical framework inspired by the work of Vygotsky. The main objective of this work is to demonstrate that technological tools can assume a crucial role in supporting teaching and learning processes if they allow teachers to reconfigure mathematical objects with the aim to create activity settings where students can interact with mathematical knowledge integrating a perceptive-motor approach to mathematical knowledge with a symbolic-reconstructive one. Re-configuring a mathematical object, which is abstract and formal, means transforming it in order to make it more concrete with the aim of using it in the student's Zone of Proximal Development, where learning can occur and teaching can be defined. In the paper two examples of application of the model will be furnished, making reference to the work of design and implementation we are developing within a European project called aITALES.
\end{abstract}

\section{INTRODUCTION}

Over recent years there has been increasingly widespread debate over the mediation role that ICT-based systems can play within mathematics education. For some time research in this field has focused either on mathematics teaching on the one hand or on mathematics learning on the other.

The experience of CAI (Computer Aided Instruction) focused on the contents to be learned and on transmission methods, assigning the computer the role of mediator in the transmission and presentation of mathematical contents and concepts.

The original version of this chapter was revised: The copyright line was incorrect. This has been corrected. The Erratum to this chapter is available at DOI: 10.1007/978-0-387-35701-0_35 
Later, criticism of the transmission approach together with the development of more advanced technologies shifted the focus towards more constructivist applications of computer technology in didactic practice. The idea of the learner-centred approach was developed: students were seen as being active players in the construction of their mathematical knowledge.

Computer assisted learning (CAL), which is still a significant element in present-day education, has focused on the construction of highly interactive systems based on microworlds. These systems incorporate a domain of mathematical knowledge that the students can explore through interaction with the primitives present in the system interface. These systems are no longer created for transmitting content, but rather for supporting student learning processes; learning is seen as the outcome of student-software interaction (Abelson \& diSessa, 1981; Papert, 1980).

The cultural-historical frameworks inspired by the work of Vygotsky highlighted the necessity within all educational activities to intertwine the teaching and learning processes (Bartolini Bussi, 1994). The link between these two processes is to be found in what Vygotsky defines as the Zone of Proximal Development (ZPD) (Vygotsky, 1978). The cultural-historical framework is centred on educational activity in its entirety. Elements of crucial importance are the use of tools and the role of the teacher: the tools are seen as mediator of the individual's actions and of the communication that takes place between participants in the activity; the teacher is seen as a co-participant in the activity who gives assistance to the students' performance.

Our work in designing mathematics teaching/learning systems falls squarely within this framework of reference, which on the one hand appears extremely promising for educational applications but on the other raises a series of research issues and questions:

- What criteria should be used in the design of mathematics learning systems to ensure that they can be used to support teaching and at the same time to mediate learning activities?

- What criteria should be used for selecting the resources for incorporation in the system, which is to foster the teaching and learning of a particular knowledge domain?

- What types of cognitive and epistemological references should be taken on board when implementing the chosen maths resources so that interface characteristics are appropriate for the students' knowledge level and for the development of significant mathematics learning?

This paper will seek to address some of these questions and will describe some of the reference works we draw on when designing and implementing systems for mathematics teaching and learning. To these ends, we will 
discuss our activity in the European project called ITALES (Innovative Teaching and Learning Environments for Schools IST-2000-26356); our involvement entails the design and development of ARI-LAB-2 (Bottino \& Chiappini, 2001), a system for mathematics teaching and learning in primary and secondary school. This system is a teaching and learning environment for primary and secondary school. In order to explain and justify the criteria and the references we draw on when designing maths systems, we will concentrate in particular on two microworlds we are developing for inclusion in ARI-LAB-2: these microworlds are currently undergoing implementation.

\section{GENERAL CRITERIA FOR DESIGNING OF ICT BASED SYSTEMS SUPPORTING TEACHING AND LEARNING PROCESSES IN MATHEMATICS}

Research in the psychology of learning has shown that, fundamentally, there are two modes of knowledge building: the perceptive-motor approach and the symbolic-reconstructive approach. The former is not limited to the initial phases of cognitive development, but is also involved in the most advanced learning processes, which includes mathematics (consider the role played by graphic representations in mathematics or that of mathematical machines for the development of specific ideas). The perceptive-motor approach involves action and perception, and produces learning based on doing, touching, moving and seeing. The second mode of knowledge acquisition is the symbolic-reconstructive approach, which is active from the very beginning of the child's cognitive development. This works with symbols (linguistic, mathematical, logical) and involves the mental reconstruction of 'objects', meanings and their mental representations. It is a more sophisticated way of knowing, one that requires awareness of procedures together with appropriation of the symbols used and of their meanings.

It is important to note that both of these approaches to knowledge acquisition are mediated by tools of a different nature, and that these carry out their mediational functions in profoundly different ways (Vygotsky, 1978). Vygotsky distinguishes between two types of mediation tool for each human activity: technical tools and psychological tools. The former mediate the interaction of the individual with the outside world, and are directed towards the control of nature. The use of such tools brings about a transformation in objects: that is, it produces external effects on the object that can be controlled at the perceptive-motor level. Psychological tools, by 
contrast, are directed toward the mastery or control of one's own processes of behaviour. They are directed internally and do not produce any transformation in the object. Vygotsky, moreover, notes that the development of the higher psychological functions involved in all processes of comprehension and of meaning construction is the result of an integration of technical and psychological tools in the activity.

The acquisition and application of technical tools, controlled by means of the perceptive-motor approach, extends the individual's sphere of influence and allows the development of new experience, while the acquisition and application of new psychological tools, controlled by means of the symbolicreconstructive approach, raises the levels of influence and awareness of the processes and phenomena which characterise human activity (Engström, 1997).

The acquisition and application of these two different types of tools occur in the student's Zone of Proximal Development (ZPD), defined by Vygotsky as "the distance between the actual developmental level as determined by independent problem solving and the level of potential development as determined through problem solving under adult guidance or in collaboration with more capable peers" (Vygotsky, 1978, p. 86).

A student's ZPD is characterized by a strict connection between teaching and learning process. Effective teaching can be defined as being within a student's ZPD.

Tharp and Gallimore give a very useful and operative definition of teaching: it consists in assisting performance through a student's ZPD. Teaching occurs when the performance of a student engaged in a task is achieved with assistance.

Tharp and Gallimore point out that assistance is best provided through instructional conversation, a dialogue between teacher and learner in which the teacher listens carefully in order to grasp the students' communicative intent, and tailors the dialogue to foster their emerging understanding (Tharp \& Gallimore, 1999).

According to Tharp and Gallimore there are different ways of offering assistance; the forms of assistance for student learning need to be accomplished by creating activity settings that maximise opportunities for co-participation and instructional conversation with the teacher and with peers.

One criterion for activity settings is that they need to permit maximum assistance in the performance of the task at hand. These settings must engage students in goal-oriented activity in which the teacher may participate as an assistant or co-participant as the need arises.

Within this framework we believe that appropriate tools can assume a crucial role in the structuring of activity settings in which assisted 
performance can be maximised; we believe also that assisted performance can be maximized if the mathematical teaching objects, which are abstract and formal objects, can be reconfigured in appropriate way in order to create activity settings where it is possible:

- to exploit a perceptive-motor approach to mathematical knowledge both during the problem solving activity and the relate instructional conversation

- to favour a strict integration between the perceptive-motor approach and the symbolic-reconstructive one during the development of the didactical activity.

Our work on designing and implementing systems for mathematics teaching and learning is based on the idea that ICT can play an important role in the reconfiguration of the mathematical objects of teaching according to the above mentioned aims.

In the following sections we will try to analyse some problems which we have taken into account in order to reconfigure mathematical objects of teaching in the domain of the rational numbers.

\section{THE CONTEXT OF APPLICATION: THE CASE OF RATIONAL NUMBERS}

In standard practice the teaching of rational numbers in lower secondary school is primarily based on the development of algorithmic and calculus skills.

Numerous investigations into mathematics teaching have highlighted that the knowledge gained by students is often lacking in stability. At best, satisfactory technical training is achieved, as well as correct use of calculation rules. In standard practice there is a marked split between the treatment of fractions on the one hand and meaning on the other. It is hard to get students to develop concepts about the properties that typify rational numbers and that distinguish them from, say, natural numbers. It is difficult to get students to develop ideas regarding concepts like the class of equivalence, of order or of density in $\mathrm{Q}$.

It thus seems that teachers experience difficulty in providing a structure for the thoughts and actions that lead to the development of sound ideas about these concepts, and this makes it difficult for students to cope with tasks that entail non-standard use of these properties.

In addition, we note that in standard teaching practice great importance is given to the symbolic-reconstructive approach; for example, graphic representation is employed only for building the concept of the fraction as an 
operator. This type of teaching approach appears unsuitable for getting students to construct appropriate meaning in this knowledge domain.

To overcome these difficulties, we have designed two microworlds, the Fractions Microworld and the Manipulator Microworld. The former can be employed for shaping an activity setting that encourages exploration of the properties of rational numbers, conjecturing about those properties, and then empirical validation.

The Manipulator Microworld can be used for shaping an activity setting that fosters the capacity to manipulate symbolically numerical expressions in $\mathrm{Q}$ so as to demonstrate the properties of rational numbers that have emerged during work with the Fractions Microworld.

Figure 1 outlines a visual model, based on Thale's theorem, that will be embedded in the Fractions Microworld in order to allow students to explore the properties of rational numbers. The model is comprised of two half-lines that have the same origin: the half-line $r$ of numbers and the half-line $t$ for performing divisions and carry-overs of selected lengths along $\mathrm{r}$.

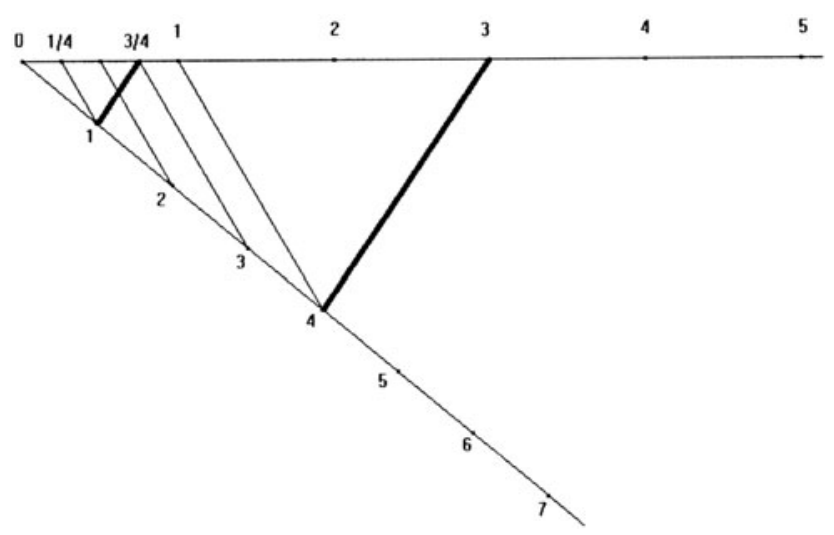

Figure 1. Visual model of the fraction microworld

Interactions with this visual model in the microworld allow the students to construct a mental image of concepts such as the class of equivalence, of order or of density in $\mathrm{Q}$. This is done by performing divisions of selected lengths along $r$, by using specific commands (for example zooming in on points along the line), by reading and interpreting the graphic effects produced when using the resources at hand.

The visual feedback of the Fraction Microworld has been studied in order to promote the construction of concepts related to rational numbers, exploiting geometrical properties that can be controlled by means of a perceptive-motor approach. 
The microworld also makes it possible to develop schemes for performing rational number operations based on division, and on carry-over, the sum and the difference of lengths selected along the half-line $r$ of numbers.

We remark also that in this microworld the perceptive-motor activity produces effects on the symbolic fraction notation. In this way meanings constructed through the perceptive-motor activity can contribute to assign a sense to the use of fraction notation.

Figure 2 shows the set of commands available in the Manipulator Microworld for performing symbolic manipulations of numerical expressions in $\mathbf{Q}$.

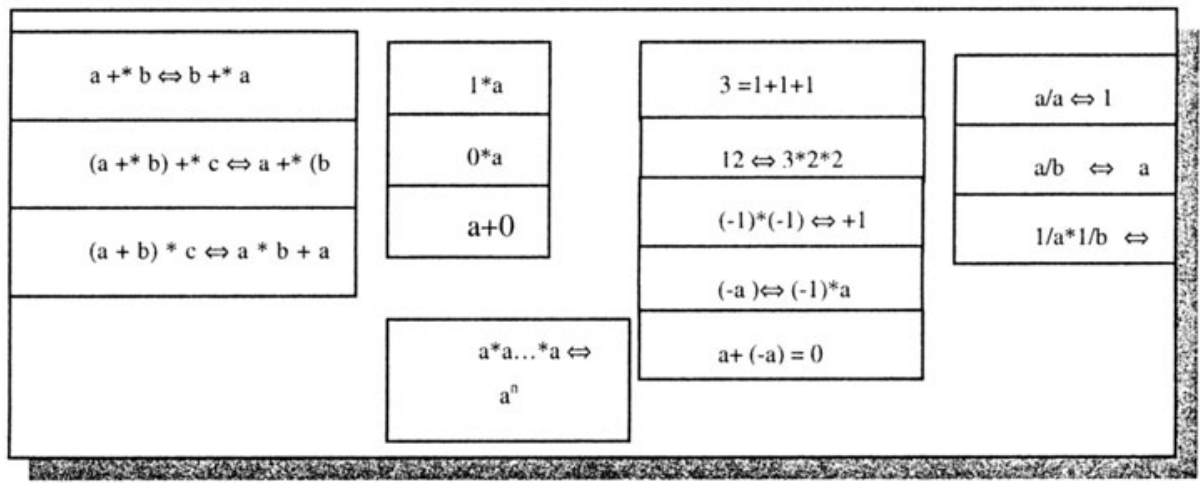

Figure 2. Command list in the interface of manipulator microworld

This set of commands has been designed for the building of an activity setting in which manipulation of numerical expressions in $Q$ is seen not as application of calculus rules but as a demonstration of the equivalence of two forms of numerical expression. This demonstration can be worked out by means of axioms reified in the interface of the system that allow the user to visualize and to make explicit mathematical entities and the relations that occur in the symbolic manipulation.

In addition it should be noted that this microworld was designed to be used together with the Fractions Microworld so as to demonstrate conjectures about the properties of rational numbers that emerge during work with the Fractions Microworld (e.g. for demonstrating that $1 / 3$ is equivalent to $1-2 / 3$, that $2 / 3+3 / 5$ is equivalent to $(2 \times 5+3 \times 2) /(3 \times 5))$.

We note that the prototype models of these two microworlds underwent two years of trials and testing in the classroom, where students used paper and pen, ruler and compass, under the guidance of highly motivated and expert teachers. The trial results highlighted the specific needs of students in problem solving, and those of teachers in providing assistance. When the 
two microworlds were used, totally new possibilities were opened up through the potential for visualisation, interaction and computation offered by the technology.

\section{CRITERIA FOR THE RE-CONFIGURATION OF THE MATHEMATICAL TEACHING OBJECTS}

In the previous section we saw that the mathematical teaching objects are not only accessible by means of a symbolic-reconstructive approach, but also starting from a perceptive-motor approach. They are objects that can be reconfigured by means of the appropriate use of specific technologies. Reconfiguring a mathematical teaching object, which is abstract and formal, means transforming it in order to make it more concrete so as to use it in the student's ZPD, where learning and teaching can occur.

The mathematical teaching object begins to come alive through the incorporation of specific 'mathematical resources' for the activity embedded within a technological tool.

Through the process of incorporation of a mathematical resource in a system an important cultural transformation is accomplished. A resource that, up to that moment, could exist only on a purely mental plane because it was accessible only through a symbolic approach, is reified and is made available in a tool with which the subject can interact, being able to observe the effects produced by the system. Let us see some examples.

The Fraction Microworld embodies the possibility of using operational schemes based on divisions of selected lengths (which can be controlled by a perceptive-motor approach) in order to relate different fractions, which are equivalent to each other, on the same point in the number line. In this way students can approach rational number concepts such as the class of equivalence of fractions by means of a perceptive-motor approach.

The Manipulator Microworld embodies the elementary properties of the arithmetical operations. These properties are incorporated in the Manipulator Microworld through the use of specific commands that can be used to demonstrate the equivalence of two numerical expressions in $\mathrm{Q}$.

When students use these commands they exploit the experience of other people who in the past have tried to resolve problems similar to those with which the student is involved. This experience is reflected in the commands available in the interface of the system.

Through this process of incorporation, the system user is provided with the operational means for tackling and solving classes of problems related to the knowledge domain that the system addresses. 
We observe that the mathematical resources embedded in a system reify mental processes involved in interaction with aspects of the system's mathematical culture of reference. Incorporation therefore creates the conditions whereby a formal, abstract knowledge domain can become an operative, concrete field of experience for both students and teachers (Boero, Dapueto, Ferrari, Garuti, Parenti \& Scali, 1995).

The framework thus far described implies that the designer of a mathematics learning system needs to tackle and solve problems that are critical to the system's educational purpose. These can be summed up in the following questions: When addressing a particular knowledge domain, which resources should be incorporated and why? How should those resources be incorporated?

We note that the answer to the first question determines the particular epistemology of mathematical knowledge that may emerge through the activity, and thus the particular type of mathematical experience that the student can develop with the system's mediation. Choosing a geometrical model based on Thale's theorem in order to approach rational numbers steers the students towards the development of a didactical practice focused on the exploration of properties of numbers, rather than the teaching of rules and algorithms of calculus.

Presenting the manipulation of numerical expressions for demonstrating equivalence between them strongly influences the kind of classroom activity the teacher can adopt and, consequently, the type of mathematical experience that students engage in with the system's mediation (Cerulli \& Mariotti, 2002).

As to the second question, we note that a mathematical resource is the expression of a system of rules that defines the socially shared process through which the resource produces an acceptable mathematical result.

At the very least, the incorporated resource expresses a single rule but it usually corresponds to a set of rules. We note that if the system has been designed to pursue efficiency and economy of thought, then the resource will probably be associated with a complex, interconnected set of rules. In such cases it may prove difficult to work back from the outcome reached and arrive at the set of rules underpinning the resource; hence, the system is opaque from the user's point of view. However, it is often educationally useful to incorporate resources in such a way as to achieve greater transparency, so that the user can recognise the rule involved on the basis of the outcome of interaction. This has been the aim behind the design of the two microworlds described in this work.

The Fraction Microworld makes available elementary resources of division, carry-over, sum and difference of lengths. They produce effects that can be easily understood by students using their spatial experience. 
Moreover, through interaction with the system, the meaning constructed using these resources can contribute to the use of fraction notation.

The Manipulator Microworld, as distinct from other comparable systems, provides the students with resources for working out elementary transformations, each of which corresponds to only one axiom. We think that this choice is particularly appropriate for students who are approaching algebraic manipulation. As a matter of fact the application of the 'commutative property' resource on a part of the expression produces an effect on the form of the expression which can be grasped at the visual level. This allows students to recognize the rule underlying the use of this axiom.

The analysis reported suggests different criteria for evaluating the efficiency brought to mathematics teaching/learning by the incorporation of resources within a system.

We believe that an effective re-configuration of the mathematical teaching objects can be realized if resources embedded in the system:

- are able to suggest to the students possible courses of action to take when solving the task at hand and/or encourage the emergence of objectives to that end;

- make it possible to validate the strategy employed in task solution and/or offer support for strategy planning that can be controlled at the perceptive-motor level;

- produce graphical and spatial effects useful for developing a mental image related to the mathematical teaching objects of reference for the system;

- produce effects on the symbolic notation that can be put into relation with graphical and spatial effects obtained through the use of resources that can be controlled at a perceptive-motor level;

- produce effects according to mathematical rules which the student can recognize and control through interaction with the system interface;

- produce effects that can, in subsequent instructional conversation between teacher and students, be interpreted as mathematical phenomena through the metaphorical use of what the interface has exhibited dynamically and concretely in the interaction.

These criteria allow us to consider possible scenarios for the use of the microworld while still in the design phase.

We think that these microworlds can contribute to structuring an activity setting that allows students to solve meaningful tasks in the domain of rational numbers and permit teachers to assist students' performance in their ZPD.

Our work does not seek to propose a generalisation from the specific domain of knowledge considered, but rather to subject our particular 
experience to a systematic and critical analysis that stimulates thinking about similar issues in other domains of mathematical knowledge.

\section{CONCLUSION}

To conclude, we believe it is important to tackle an important research issue regarding the relationship between the tool and the construction of meaning, which is a subject of international debate (Jones, 1998).

The cultural-historical framework considers a tool as the result of cultural evolution, produced for specific aims and consequently incorporating an idea. That does not imply that the tool itself is the source of meaning or that, at the didactic level, meaning emerges from the interaction between the student and the tool. Rather, meaning resides in the purposes for which tools are used, in the strategies that gradually take shape in the course of purposely designed activity undertaken with those tools.

We think that the meaning resides not in the tools but in the aims for which the tool is used; in the plans that are developed for using it. These plans, expressed in socially shared language, gradually realised in the course of the activity with the tool, constitute the meaning, in that they reflect the course of the activity mediated by the tool and orientated toward an aim.

Accordingly, the key element in the construction of meaning is the type of activity setting that the teacher creates and manages, so as to maximise opportunities for technology-mediated co-participation and instructional conversation between teacher and student and among the students themselves mediated by technologies.

In this paper we have addressed a vital aspect of the construction of an activity setting, namely the reconfiguration of teaching objects via suitable technologies. However, there is another aspect to be considered, and that is the 'reconfiguration' of teachers, so that they are able to make full use of the potential offered by the tools available in the learning process. This is a matter of crucial importance; unless it is solved, computer technology (no matter how important and advanced) will fail to bring about any innovation whatsoever in teaching practice.

\section{REFERENCES}

Abelson, H. \& diSessa, A. (1981). Turtle Geometry: The Computer as a Medium for Exploring Mathematics. Cambridge: MIT Press.

Bartolini Bussi, M. G. (1994). Theoretical and empirical approaches to classroom interaction. In R. Biehler, R. W. Scholz, R. Strasser \& B. Winkelmann (Eds), Didactics of Mathematics as a Scientific Discipline. Dordrecht: Kluwer. 
Bottino, R. M. \& Chiappini G. (2002). Advanced technology and learning environments: Their relationships within the arithmetic problem-solving domain. In L. D. English (Ed.), Handbook of International Research in Mathematics Education (pp. 757-786). London: Lawrence Erlbaum \& Associates.

Boero, P., Dapueto, C., Ferrari, E., Garuti, R., Parenti L., \& Scali E. (1995). Aspect of the mathematics-culture relationship. Proceedings of PME XIX Recife (Vol. 1, pp. 151-166). Brazil: Universidade Federal de Pernambuco.

Cerulli, M. \& Mariotti M. A. (2002). L'algebrista: Un micromonde pour l'enseignement et l'apprentissage de l'algèbre sciences et techniques éducatives. In J. F. Nicaud, E. Delozanne \& B. Grugeon (Eds), Logiciels pour l'Apprentissage de l'Algèbre Nicaud (Vol. 9, No. 1-2, pp. 149-170).

Engström, Y. (1987). Learning by Expanding. Helsinki, Finland: Orienta-Consultit.

Jones, P. (1998). Tools, Symbols and Ideality in Ilyenkov ISCRAT (International Society for Cultural Research and Activity Theory). Aarhus: Denmark.

Papert, S. (1980). Mindstorms. Brighton, Sussex: Harvester Press.

Tharp, R. \& Gallimore R. (1989). Rousing Minds to Life. Teaching, Learning and Schooling in Social Context. Cambridge, MA: Cambridge University Press.

Vygotsky, L. S. (1978). Mind in Society: The Development of Higher Psychological Processes. Cambridge, MA: Harvard University Press. 